\title{
Intermediary networks under the rule of equi-repartition of profits
}

\author{
Fabien Mercier ${ }^{1}$ \\ Université Panthéon-Assas, France \\ Email: Fabien.mercier@yahoo.fr
}

Received: 1 June 2015 / Revised: 9 September 2015 / Accepted: 14 September 2015 / Published online: 16 November 2015

\begin{abstract}
This paper studies the stability, equilibrium and efficiency of directed networks of trade intermediaries under a rule of equi-repartition of profits. This equi-repartition rule, although simple and natural, introduces complex topologic considerations from players. Core-periphery structures can arise endogenously and result in stable networks even assuming complete homogeneity of all agents in the network. The concept of network partition is introduced to provide examples of networks that could be seen as arising from the linking up of a set of disjoint networks in an endogenous manner. From an aggregate welfare perspective as well as sometimes from an individual perspective, leaving the linking up of those individually efficient networks to each individual agent can result in an inefficient aggregate network, even if taken in isolation each pre-existing network is efficient.
\end{abstract}

\section{JEL classification: D85, G10, L14}

Keywords: trading; telecommunications; settlements; post-trading industry; networks; graph-theory.

\section{INTRODUCTION}

Network economics modelling has been successfully applied over the last decade to describe economic phenomenon as diverse as the social transmission of job information (Calvo-Armengol and Jackson), free-trade agreements (Goyal and Joshi), and co-authorship links (Jackson and Wolinsky). Network economics have also provided models that are phrased in general terms and can thus potentially be applied to a variety of situations (see for example Bala and Goyal, Belleflamme and Bloch, Dutta and al, Goyal and Vega-Redondo, Jackson). Concerns about the financial resilience of the banking system has led to a renewed interest in the modelling of the complex links between financial institutions. Of particular importance are networks of intermediaries related to trading between banks and financial institutions. Recent empirical evidence suggests that these trading relationships follow a core-periphery structure, whereby a group of banks in the core are densely linked to each other and a set of peripheral banks maintain links with banks from the core, but not between each others (Craig, Veld, Fricke). In this paper

\footnotetext{
1 Corresponding author: Laboratoire d'Economie Mathématique et de Microéconomie appliquée (EA4442), 4 rue Blaise Desgoffe, 75006 Paris, Phone: 33 (0) 144415787 , fabien.mercier@yahoo.fr
} 
we define a model of trade network with intermediaries, similar to Babus, Veld, and Goyal, in which each intermediary along an intermediary chain used to achieve a trade gets an equal share of the total profit derived from the overall trade, after deducing the relevant costs incurred. This profit sharing rule is consistent with Siedlarek bargaining model in the case where the time available to all parties of the transaction to bargain down the fees of intermediaries tends to 0 . Goyal and Vega-Redondo allow intermediation between players, but in the opposite limiting case of Siedlarek bargaining model where the time available to all parties of the transaction to bargain down the fees of intermediaries tends to $+\infty$. In this opposite case, intermediaries for which there exist alternative intermediation paths cannot derive any profit from their intermediation service. This leads to the star network being the only stable network at equilibrium. Babus provides a more evolved model for the inter-bank market, but also find as main outcome the star network with a single intermediary between all players. Veld manages to obtain core-periphery stable networks, but at the cost of heterogeneous parameters for core and non-core banks. Other attempts to provide different equilibrium structures have often resulted in (unrealistic) regular networks, where all agents have (almost) the same number of links.

The first main contribution of this paper is to show how a rule of equi-repartition of profits endogenously leads to stable core-periphery networks, without having to resort to any heterogeneity assumptions concerning the network, contrary to. Although most of the results derived in this paper concern the case where only one strict intermediary is allowed to complete a trade, similar to and most of the papers mentioned above, this particular result of stable coreperiphery network is extended to the case of intermediary chains of arbitrary length. The family of core-periphery networks obtained also allow for very different number of links for each agent in the core.

The second main result of this paper is to show how core-periphery networks can result from the linking up of disjoint structure that are, when considered individually, efficient, while failing to achieve global efficiency of the aggregate network. Policy implications are that there can be benefit in having a central planner or providing additional incentives to agents for making them re-arrange the aggregate network to reach more efficient outcomes.

Section 2 presents standard graph-theoretic notions used in the rest of the paper and defines the family of games studied. Section 3 characterises parameter constellations for which the most usual structures, such as the stars, are stable networks, with a view to better understand the strategic considerations of agents in the intermediation networks defined. Section 4 introduces the notion of network partition in order to formalise the types of structures that are stable yet contain a given set of subnetworks, and to construct concrete examples of core-periphery networks arising from the linking up of stars, for which we coin the term of "multistars". The section then studies the efficiency of multistars as a function of these networks structural and cost parameters and provides the two main contributions of the paper mentioned above. Section 5 concludes. Proofs that are more technical are relegated to the Annex for clarity, while easier proofs remain in the main text.

\section{DEFINING A NEW FAMILY OF GAMES}

\subsection{General graph theoretic definitions}

Agents form the set of nodes $V(G)$ of a network $G$. The cardinality of a network is by definition the cardinality of its set of nodes, that we often denote by $n$. An edge, or link, is any (ordered) pair of nodes $(A, B)$, with $A$ and $B$ in $V(G)$. It is denoted $A B$. Note that the order is important, because $A B$ is different from $B A$ if $A$ and $B$ are not the same node.

Any edge $A B$ is either in the network, that is, belongs to the set of edges $E(G)$ of the network $G$, or not in the network, that is, does not belong to the set of edges $E(G)$ of the network $G$. 
An edge $A B$ from the node $A$ to the node $B$ represents some (unidirectional) "link" the agent $A$ has established with agent $B$. For example, if one thinks of the nodes as the Central Securities Depositories (CSD) of the post-trading industry, an edge between $A$ and $B$ could mean that $A$ is an investor agent in $B$ idem est holds an account with $B$ that allows it to settle transactions of securities in the domestic market of $B$. Note the relation does not need to be symmetric, that is, $A$ can have an account with $B$ (and thus be able to settle trades for its clients in the domestic market of $B$ ) without the converse being true ( $B$ does not need to have an account with $A$ ). This is the reason why the pairs are ordered, and the network is said to be directed.

As an example, consider the hypothetical network on Figure 1. Note that while some pairs of nodes are linked in both directions $(A B$ and $F E)$, other links are just one directional.

Figure 1.

A network with six nodes and 14 edges

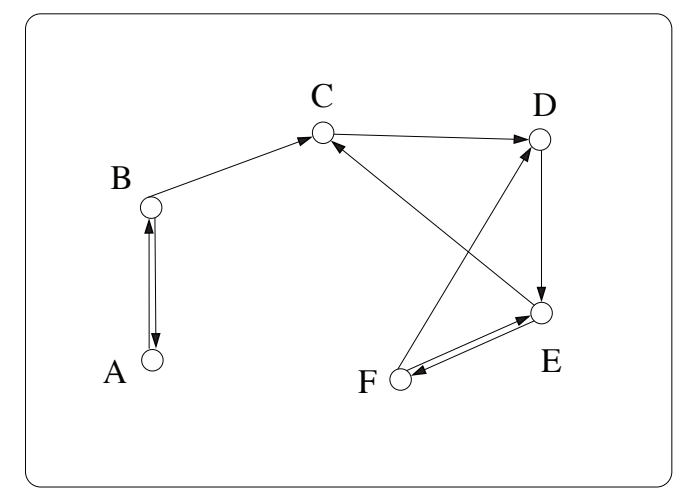

Three networks have a particular significance when studying trade or settlement networks: the empty network, which is a network with no link which generally reflects too high costs for establishing a link compared to the expected profits derived from it, the complete network, which contains all possible links and which indicates the highest desire for disintermediation, and the complete star network, where one node, the center of the star, is linked in both directions to all other nodes, the leaves of the star. Hence any trade in a complete star network involves the center of the star. Figure 2 depicts these three particular types of networks.

\subsection{Defining intermediation paths and payoffs of the games $\mathcal{G}_{k_{0}}^{n}(w, c, g)$}

For each edge $A B$ there is a cost $w_{A B}$ to build the edge $A B$, and a cost $c_{A B}$ to use it in an intermediation chain. For each pair of vertices there is a latent intermediation profit $g_{A B}$ that can be realised and shared if there is at least one admissible intermediation path from $A$ to $B$. We make these notions more precise in this section.

Figure 2.

A complete star with 6 leaves, the empty network on 5 nodes and the complete network on 4 nodes.
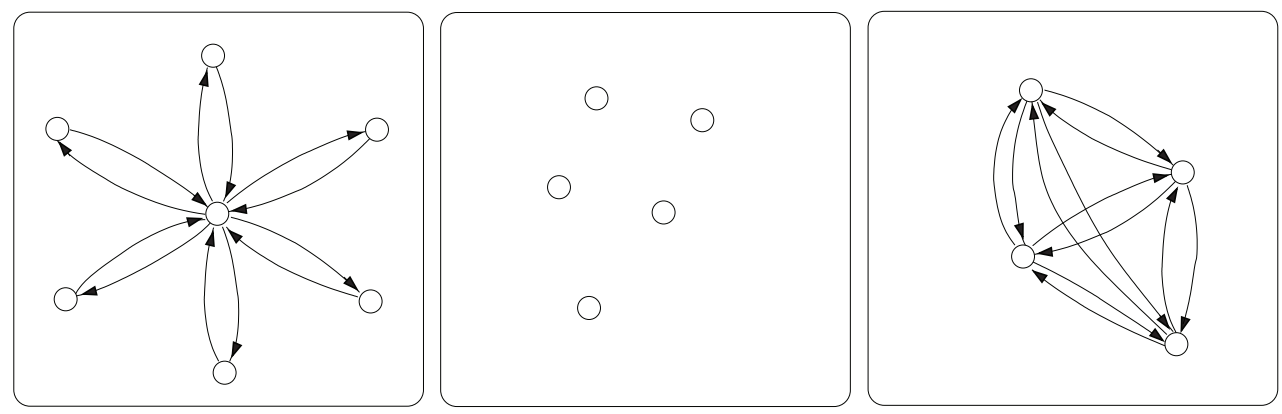
Definition 1 A path is a network $P$ whose nodes can be labelled $V(P)=\left\{u_{1}, u_{2}, \ldots, u_{k}\right\}$ such that its set of edges is precisely $E(P)=\left\{\left(u_{1}, u_{2}\right), \ldots,\left(u_{k-1}, u_{k}\right)\right\}$. We often write $P=u_{1}, u_{2}, \ldots, u_{k}$ in this case, and say that $P$ is a $u_{1} u_{k}$-path, or a path from $u_{1}$ to $u_{k}$. The length of $P$ is $k-1$, while its cardinality is $k$.

Note that our notion of path has a direction: a path from $u$ to $v$ is not a path from $v$ to $u$, at least when $u \neq v$.

Definition 2 Let $G$ and $H$ be two networks. Then $H$ is a subnetwork of $G$ if, and only if, $V(H) \subseteq V(G)$ and $E(H) \subseteq E(G)$.

Definition 3 A path of a network $G$ is a path that is a subnetwork of $G$.

Let $n$ and $k_{0}$ be two positive integers, $N$ a set of nodes of cardinality $n$ and $w, c$, and $g$ real-valued functions defined on the cartesian product $N^{2}=N \times N$. Players are the nodes in $N$ and decide to link, or not, to other nodes. Hence the action set of a given player $u$ is the set of edges $(u, v)$ for $v \in N, v \neq u$. If node $u$ establishes the link $(u, v)$ to node $v$ it has to bear a cost of $w(u, v)$.

Some paths of the resulting network can be used to carry potential trades that benefit all the players along those paths. More precisely, for each ordered pair $(u, v)$ there is the potential for a trade of value $g(u, v)$ to be realised. For the trade to be realised there must be a path of length less than $k_{0}$ in the network. A cost $c(u, v)$ is associated to each edge $(u, v)$ of the network. The cost of using a path is simply the sum of the costs of all its edges. For each pair $(u, v) \in N^{2}$ a $u v$-path of minimum cost can be selected for the trade if it is an admissible path, that is, a path of length not more than $k_{0}$ and whose costs do not exceed $g(u, v) .{ }^{2}$ If more than one such admissible path of minimum cost exist then the path used to perform the trade is selected randomly among all admissible paths of minimum cost, the uniform probability distribution being used. Each node of the selected admissible $u v$-path of minimum cost $u=u_{1}, u_{2}, \ldots, u_{k}=v$ receives an equal share of the aggregate net profit $g\left(u_{1}, u_{k}\right)-\left(c\left(u_{1}, u_{2}\right)+c\left(u_{2}, u_{3}\right)+\ldots+c\left(u_{k-1}, u_{k}\right)\right)$ derived from the trade, that is:

$$
\frac{g\left(u_{1}, u_{k}\right)-\left(c\left(u_{1}, u_{2}\right)+c\left(u_{2}, u_{3}\right)+\ldots+c\left(u_{k-1}, u_{k}\right)\right)}{k}
$$

The player $u_{i}$ are called the intermediaries of the trade. The two players $u_{1}$ and $u_{k}$ are its final intermediaries, while players $u_{2}, \ldots, u_{k-1}$ are its strict intermediaries. Players build edges such as to maximise their expected payoff function.

Theoretical justification of the equal profit splitting allocation rule can be found in Siedlarek. Indeed, in the author model of stochastic bargaining involving different chains of intermediaries, when the discount factor of the bargaining model tends to 0 , that is, when players do not have sufficient time to bargain over the splitting of profits without missing the trade opportunity (and thus become very impatient), the expected payoff of each intermediary become equal ${ }^{3}$. We are

\footnotetext{
Deleting this assumption would result in a different still interesting problem, where the nodes of the network are actually obliged to perform the transaction, even in case they take a net loss.

Defining the profit rule such that the first intermediary on a chain of intermediaries derives all the benefit from a given trade gives rise to a simpler, yet also interesting, problem. In such a setting, where node $A$ earns the whole profit derived from settling the trade of $g-k w$ when at the beginning of an intermediation chain of length $k$, one can easily prove that:

1) if $w \geq g-c$ then the empty network is a NE, and that it is the only NE if we assume further that $w \geq g-c+(g-2 c)(n-2)$

2 ) the complete network is the only strict NE if, and only if, $c>w$.

$3)$ a directed cycle is a NE if, and only if, $g-c+g-2 c+\ldots+g-(n-1) c>w$.
} 
taking this view here; the opposite view would be to assume agents have plenty of time to bargain before moving on to carry out the trade; in such a case, according to, intermediaries which are not "essential" - meaning there exists, in the network, another chain of intermediaries to which they do not belong - would earn a profit of zero.

Throughout this paper we will assume that $w, c$, and $g$ are constant functions. Hence the above profit simply reduces to

$$
\frac{g-(k-1) c}{k}=: \pi_{k}
$$

To write down players' payoffs explicitly under this hypothesis it is useful to define a minimum path, as minimum path correspond to path of minimum costs:

Definition $4 \mathrm{~A}$ minimum $u v$-path is a path from $u$ to $v$ of minimum cardinality. The set of all minimum uv-paths is denoted by $\mathcal{P}_{u v}$, and the length of any minimum uv-path by $d(u, v)$ and called the distance from $u$ to $v$; if there is no path from $u$ to $v$ then we set $d(u, v):=+\infty$. For any positive integer $k$, the subset $\mathcal{P}_{u v}^{\leq k}$ of $\mathcal{P}_{u v}$ is the set of minimum $u v$-path of cardinality less or equal than $k$. We also set $\mathcal{P}_{u v}^{\leq \infty}:=\mathcal{P}_{u v}$ for convenience.

Definition 5 A minimum path is a path such that there exists two nodes $u$ and $v$ such that it is a minimum uv-path. We denote by $\mathcal{P}$ the set of all minimum paths of the network. Hence, $\mathcal{P}=\underset{\{u, v\} \subseteq V(G)}{\cup} \mathcal{P}_{u v}$.

Since the cost function is constant, the set $\mathcal{P}_{u v}$ of minimum $u v$-path is precisely the set of paths of minimum costs, from $u$ to $v$ and the set $\mathcal{P}_{u v}^{\leq k_{0}}$ of minimum $u v$-path of cardinality less or equal than $k_{0}$ is precisely the set of admissible $u v$-paths. The full expression of the payoff function for agent $i$ of the network in the game $\mathcal{G}_{k_{0}}^{n}(w, c, g)$ can then be expressed as:

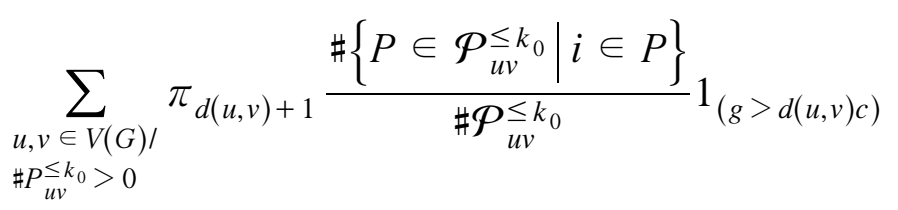

We will always denote by $k_{0}$ the maximum number of intermediaries allowed in the rule of the game, and set $k_{0}=+\infty$ if chains of intermediaries of arbitrary length are allowed. Note that for any given game with $n$ players, allowing an infinite number of intermediaries is equivalent to allowing at most $n$ intermediaries. That is, for all $n$, we have: $\mathcal{G}_{\infty}^{n}(w, c, g)=\mathcal{G}_{n}^{n}(w, c, g)$. Also note that in the formula giving agent $u$ 's profits we always have $d(u, v) \in\left\{1,2, \ldots, k_{0}\right\}$ for all pairs of nodes $(A, B)$ which appear in the sum. Finally, because paths of cardinality $k$ such that $g<(k-1) c$ are not admissible, we have that for all $n, \mathcal{G}_{k_{0}}^{n}(w, c, g)=\mathcal{G}_{\min \left(k_{0}, \frac{g}{c}+1\right)}^{n}(w, c, g)$. Hence we can

\footnotetext{
3) the star is a NE if, and only if, $c \leq w \leq g-c$

4) If $-w+g-c>0$ then any NE is strongly connected, with diameter $\Delta<w / c+1$

5) In a NE having more than two components, all vertices earn a 0 payoff.

Point 5) involves a very easy argument. Indeed, assumes $G$ is a NE with at least two different components, with $A$ and $B$ two nodes belonging to different components of $G$ and with $B$ earning a non-zero payoff $\pi_{B}>0$. Because $A$ is not in the same component of $B$ it does not derive any profits from trades with the vertices $B$ is trading with. $A$ can thus strictly increase its profit by $\pi_{B}$ by simply building an edge to each of the out-neighbours of $B$, a contradiction with NE. Notice this argument is not valid anymore if all intermediaries share profits, since the higher payoff of $B$ maybe due to its strategic intermediation role between vertices of its own component, and $A$ cannot replicate $B$ role in that matter as it cannot replicate the edges $C A$ for $C$ in-neighbour of $B$. It is this more complex environment that we investigate in the next section.
} 
assume without loss of generality that $k_{0} \leq \frac{g}{c}+1$. This allow to drop the factor $1_{\left(g>\left(n_{A B}-1\right) c\right)}$ in the expression of players' profits.

Definition 6 A Nash equilibrium (resp. a strict Nash equilibrium) is a strategy profile $G$ in which any agent deviating unilaterally would not be better off (resp. would be worse off).

Because this payoff function takes into account the overall structure of the network by making the set of all minimum paths containing $u$ a pre-requisite for computing $u$ 's profits, it can be very difficult to characterise all nodes' strategies that result in a Nash equilibrium. Indeed, because from a given strategy a unilateral deviation from a given player consists in building or deleting edges starting at $u$ at the same time, thus reshaping the set of all minimum paths in a way difficult to predict, we will often resort to the more general notion of stability:

Definition 7 A network $G$ is stable (resp. strictly stable), if, and only if, adding or deleting any single edge $(u, v)$ would not increase agent u's profits (resp. strictly increase agent u's profits).

This would translate the idea that given an initial network, each node in turn only considers one edge at the time, computing whether it would be better off by severing it if this edge is in the network or by creating it if it is not in the network. In a stable network no node would add or delete an edge.

Proposition 8 If $G$ is a Nash equilibrium (resp. strict Nash equilibrium) then it is stable (resp. strictly stable).

The proof is trivial and follows from definitions. Hence establishing some necessary properties of stable networks certainly establishes the same properties for the more restricted class of Nash equilibrium networks. The converse need not be true, although stable graphs which are not Nash equilibria are not straightforward to construct. The question of defining a family of stable graphs which are not Nash equilibria is thus left open.

Definition 9 A network is strongly connected if, and only if, for any pair $(u, v)$ of its nodes, there exists a path going from node u to node $v$.

Definition 10 The diameter $\Delta$ of a network is defined by $\Delta=\max \{d(u, v), u \neq v\}$.

Definition 11 Let $G$ be a network and $A$ be a node of $G$. We denote by $N^{+}(A)$ the set of all nodes towards which A maintains a link, and by $N^{-}(A)$ the set of all nodes which maintain a link towards $A$. The cardinality of $N^{+}(A)$ is called the out-degree of $A$, and is denoted $d^{+}(A)$, while the cardinality of $N^{-}(A)$ is called the in-degree of $A$, and is denoted $d^{-}(A)$.

Definition 12 A graph $G$ is d-regular if all its nodes have both in and out-degree equal to $d$. A graph $G$ is regular if there exist a natural number $d$ such that $G$ is d-regular. 


\section{RESULTS}

\subsection{Bounds to the diameter}

The diameter of a network is thus the maximum distance between any two nodes of the network. Note that the diameter of a network is finite if, and only if, the network is strongly connected.

Proposition 13 If $w \geq \frac{g-c}{2}$, then any stable network is strongly connected and its diameter satisfies $\left.\Delta \leq \min \left(k_{0}-1, \mid \frac{\frac{g+c}{2}+w}{\frac{g+c}{2}-w}\right\rfloor\right)$. If (i) $w<(g+c) \sum_{k=2}^{n} \frac{1}{k}-c$ and (ii) $k_{0}-1 \geq\left\lfloor\frac{\frac{g+c}{2}+w}{\frac{g+c}{2}-w}\right\rfloor$, then the bound is optimal, with the directed circle C of diameter $\Delta_{C} \leq\left\lfloor\frac{\frac{g+c}{2}+w}{\frac{g+c}{2}-w}\right\rfloor$ being a stable
network for which the bound is reached.

The proof of the bound validity is provided in Annex 6. The stability of the directed circle of the mentionned diameter is easy to prove by symmetry.

Notice in passing that another (weaker) necessary condition for the minimum path to be able to carry out a trade is that $g-\Delta c>0$ ie $\Delta \leq g / c$. Hence the above results also contains the somehow simpler condition that $\Delta \leq g / c$.

\subsection{Extreme cases: no links, or all the links}

\subsubsection{The empty network}

Proposition 14 If $w \leq \frac{g-c}{2}$ then the empty network is a Nash equilibrium.

Proof. If any player deviates unilaterally from the strategy profile yielding the empty network, it means it has built some number $k$ of edges. But building such edges incurs a cost of $k w$, and a profit of only $k \frac{g-c}{2}$. Since $k \frac{g-c}{2}-k w \leq 0$, this player is not better off.

Clearly, if a strict inequality holds, the empty network becomes a strict Nash equilibrium.

Note that if $\frac{g-c}{2} \leq w<g-c$ deviating would be socially strictly more efficient. Indeed, the aggregate profit would be of $g-c-w>0$.

Remark 15 This proposition does not imply that the empty network is the only Nash equilibrium. For example, assume $g>2 c$ and $\frac{g-c}{2} \leq w<\frac{g-c}{2}+\frac{g-2 c}{3}$. Then the circle of cardinality three is a strict Nash equilibrium, as well as the empty network.

Nevertheless we have the following result:

Proposition 16 If $w>\frac{g-c}{2}+2(n-2) \frac{g-2 c}{3} 1_{\left(k_{0}>2\right)}+(n-2)(n-3) \frac{g-3 c}{4} 1_{\left(k_{0}>3\right)}=: l_{0}$, then the empty network is the only strict stable network (and the only strict Nash equilibrium).

The proof is provided in Annex 6 . 


\subsubsection{The complete network}

Proposition 17 If $w<\frac{g+c}{6}$, then the complete network is the unique strict stable network (and it is a strict Nash equilibrium).

Proof. Suppose $w<\frac{g+c}{6}$ and let $G$ be a stable network. First, since $\frac{g+c}{6}<\frac{g-c}{2}$ we have that $w<\frac{g-c}{2}$ and thus by Proposition 13 the network is strongly connected. We then prove a property called transitivity: if $(A, B)$ is in the network and $(B, C)$ is in the network then $(A, C)$ is in the network. Together with strong connectivity it can easily be checked that it implies the network $G$ is the complete network.

Let $(A, B)$ and $(B, C)$ be two edges in the network. Assume by contradiction that the edge $(A, C)$ is not in the network. Then the additional profit for $A$ to build edge $(A, C)$ is equal to the sum of the additional gains from trades of type $(A, C)$ and of the intermediation fees from all new minimum paths using $A C$, minus the cost $w$ of building $A C$. Since the additional gains from being able to settle trades with $C$ directly (without using $B$ as an intermediary) are $\frac{g-c}{2}-\frac{g-2 c}{3}>w$, the node $A$ is willing to create the edge $(A, C)$, a contradiction with stability. Hence the edge $(A, C)$ was already there. This concludes the proof.

\subsection{Intermediate cases}

The following results single out the complete star as being a remarkable structure, since it is always a Nash equilibrium when the costs of building new edges are neither too high, nor too low, and this no matter the length $k_{0}-1 \geq 2$ of the intermediary chains allowed. Again, there may exists other Nash equilibriums, like the triangle mentioned in Remark 15.

\subsubsection{Characterisation of stable and Nash equilibrium complete stars}

Proposition 18 Assume $g>2 c$. A complete star of size $n$ is a stable network if, and only if,

$$
\frac{1}{6}(g+c) \leq w \leq \frac{g-c}{2}+(n-2)\left(\frac{g-2 c}{3}\right)
$$

Proof. a) No leaf is strictly better off creating a new link if, and only if

$$
\frac{g-c}{2}-w-\frac{g-2 c}{3} \leq 0
$$

b) Nor to delete one, if, and only if,

$$
w \leq \frac{g-c}{2}+(n-2)\left(\frac{g-2 c}{3}\right)
$$

c) The center does not want to delete a link, if, and only if,

$$
w \leq \frac{g-c}{2}+(n-2)\left(\frac{g-2 c}{3}\right)
$$

which happens to be the same condition as for a leaf not to delete a link. 
The inequality, if strict, characterises stars that are strict Nash equilibrium.

Interestingly, the condition for a complete star to be a stable network (resp. strict stable network) is the same as for being a Nash equilibrium (resp. strict Nash equilibrium):

\section{Proposition 19 Assume $g>2 c$. Then stable complete stars are also Nash equilibria.}

The proof is provided in Annex 6.

Notice that, holding all parameters except $n$ constant and making $n$ tend towards infinity, there exists a threshold from which the complete star becomes a Nash equilibrium. This translates the fact that when the number of agents in the economy increases, it becomes more useful to have a single intermediary (and pay to it fees for its intermediation services) than to establish connections towards each individual agent.

Remark 20 This does not imply that a subnetwork or a induced subnetwork of a network which is a star will be stable if it satisfies this condition, since strategic considerations involving nodes outside the star may dominate. For example, if a star is a subnetwork of a bigger network and some leaf $A$ of the star is linked to many vertices outside the star, another leaf B of the star may want to establish a direct link to A to derive higher profits from trades with those out-neignboors of $A$ which are not in the star (avoiding the center of the star as an intermediary for such trades). Hence, the concept of stability of a network does not translate easily to its subnetworks.

We now turn our attention specifically to the case where only a maximum of three intermediaries is allowed. Although such an assumption is clearly restrictive, this model will still capture the dependency of a node's strategic choice relatively to the overall network topology. Hence, the three intermediary case sheds some light on the nature of strategic interactions better.

As previously shown, for some constellations of parameters the complete star, the complete network or the empty network are stable networks or Nash equilibria. Nevertheless, to characterise all stable networks or Nash equilibria for any constellation of parameters is still an open question even in the three intermediary case.

\subsubsection{Characterisation of stable $d$-regular uninetworks}

We saw that when the cost of establishing an edge $w$ is neither too high nor too low, the complete star is always a stable network and a Nash-equilibrium. The complete star exhibits two striking structural features. First, in a star any two nodes are linked only by a unique path, hence there is no other admissible path for agents to carry out any given trade than this single path. Second, the star shows a singular asymmetry: the center has to sustain the costs of all links towards the other vertices of the star, and derives benefits from all the trades of the network, whereas the leaves have only to pay for a single link, but are the strict intermediaries of no trade. This is reflected at the level of individual nodes by a very high degree asymmetry, with the center being of the highest possible in and out-degree and the leaves being of in and out-degree 1, the lowest possible in and out-degree in a connected graph.

A natural question is thus to ask if a more "balanced" structure - by which we mean more symmetry in the underlying network - would necessarily provide alternative paths for dealing with a same given trade. Can symmetric networks be stable without providing for more than one alternative paths to carry out trades?

More precisely, when parameters are homogenous, game theory often studies symmetric equilibria, that is, equilibria where all players play the same strategy. This is because this approach 
is consistent with not favouring one agent more than another in predicting which network will emerge from the game ${ }^{4}$.

A symmetric equilibrium results in a vertex-transitive graph, that is, a graph $G$ for which, for any players $i, j$ there exists a graph automorphism $\sigma$ of $G$ such that $\sigma(i)=j$. Hence, both $i$ and $j$ are indeed playing exactly the same action with respect of the other nodes once a proper relabelling of the names of the players has been applied. Symmetric equilibrium thus result in (often excessively) "balanced" networks. In particular, these networks are $d$-regular, since it is easy to check that all their nodes will have both in and out-degree $d$.

Because we believe the notion of symmetric equilibrium is actually too strong, in what follows we will actually study the more general case of regular networks (some regular networks do not arise from symmetric strategies, but from other, less stringent, strategies). Hence, the question is: does there exist regular network which are stable and do not provide with alternative paths to settle trades?

Given a game $\mathcal{G}_{k_{0}}^{n}$, that is, a maximum number $k_{0}$ of intermediaries allowed, a uninetwork is a network such that each trade $(A, B)$ has precisely one corresponding admissible path. For example, in the case of $\mathcal{G}_{3}^{n}$, that is, when a maximum of three intermediaries is allowed, a uninetwork is such that for any trade $(A, B)$ either $A$ is directly linked to $B$ or there exists a unique intermediary $C$ such that $(A, C)$ and $(C, B)$ are in the network. Consequently the structures depicted in Figure 3 cannot be subnetworks of any uninetwork of a game $\mathcal{G}_{3}^{n}$, and, by extension, of any uninetwork of $\mathcal{G}_{k}^{n}$ for $k \geq 3$.

Figure 3.

Forbidden subnetworks in uninetwork $\mathcal{G}_{3}^{n}$

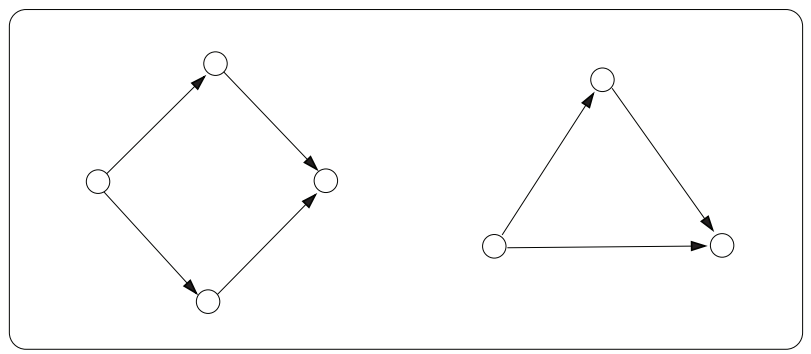

In our model, we do not require a given node $A$ to possess a closed path towards itself to carry out a trade from itself to itself. Nevertheless, to use results from algebraic graph theory, we need to make this a requirement here, that is, we need to ask that in a uninetwork there is precisely one single admissible path from any node $A$ to any node $B$ with possibly $B=A$. Admittedly, this imposes more structure than should have been the case, but this additional requirement enable us to use the classic algebraic result that the $(i, j)$ th entry of the $k$ th power of the adjacency matrix of a graph gives the exact number of walks from node $v_{i}$ to node $v_{j}$. We use one of the main theorem of Gimbert to derive a necessary condition for a regular uninetwork to be stable. For the games $\mathcal{G}_{k}^{n}$ with $k \geq 4$, the inexistence of stable regular uninetwork is straightforwardly implied by a result from the literature that there exists no regular uninetwork with a unique path of length at most $l$ for $l \geq 3$ between any two vertices.

Definition 21 Given a network $G$ the line-network of $G$ is the network $L G$ with set of nodes $V(L G)=E(G)$, the set of edges of $G$, and where a node $(A, B)$ of $V(L G)$ is linked to a node $(C, D)$ of $V(L G)$ if, and only if, $B=C$ in $G$.

Although, as mentioned in the introduction, the realism of those networks can be questionned, they are still worth studying. 
Theorem 22 [Gimbert] Let $d \geq 2$. There exists a unique d-regular network for which there exists a unique path of length 1 or 2 between any two distinct nodes and a unique closed path of length 2 from any given node to itself: it is the line network $L K_{d+1}$ of the complete network $K_{d+1}$.

Figure 4 illustrates such a network for $d=2$ :

Proposition 23 Assume $g>2 c$ Then a d-regular network is stable if, and only if,

$$
2 d\left(\frac{g-2 c}{3}\right)+\left(\frac{g+c}{6}\right) \leq w \leq \frac{g-c}{2}+2 d\left(\frac{g-2 c}{3}\right)
$$

The proof is provided in Annex 6.

Hence, although there exist stable regular networks which fail to provide more alternative paths for carrying out any given trade, the number of those networks is very limited: by Gimbert Theorem (Theorem 22), they are the line graphs of complete graphs, and by Proposition 23, only the constellation parameters satisfying the inequality stated in Proposition 23 are stable.

In conclusion, more symmetry in network structures undoubtedly favour the existence of alternative paths to settle trades, and the exceptions to this rule are few and fully caracterised in this section.

Figure 4.

The unique regular uninetwork of degree 2

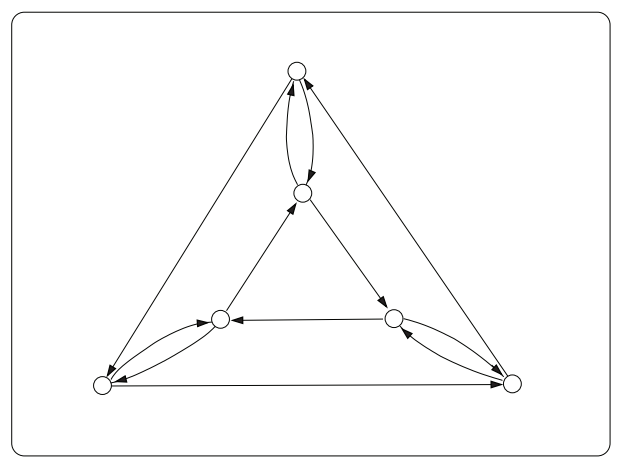

\subsection{Summary of Nash equilibria and stable networks}

Figure 13 summarises the results obtained so far.

Figure 5.

Summary of proven results

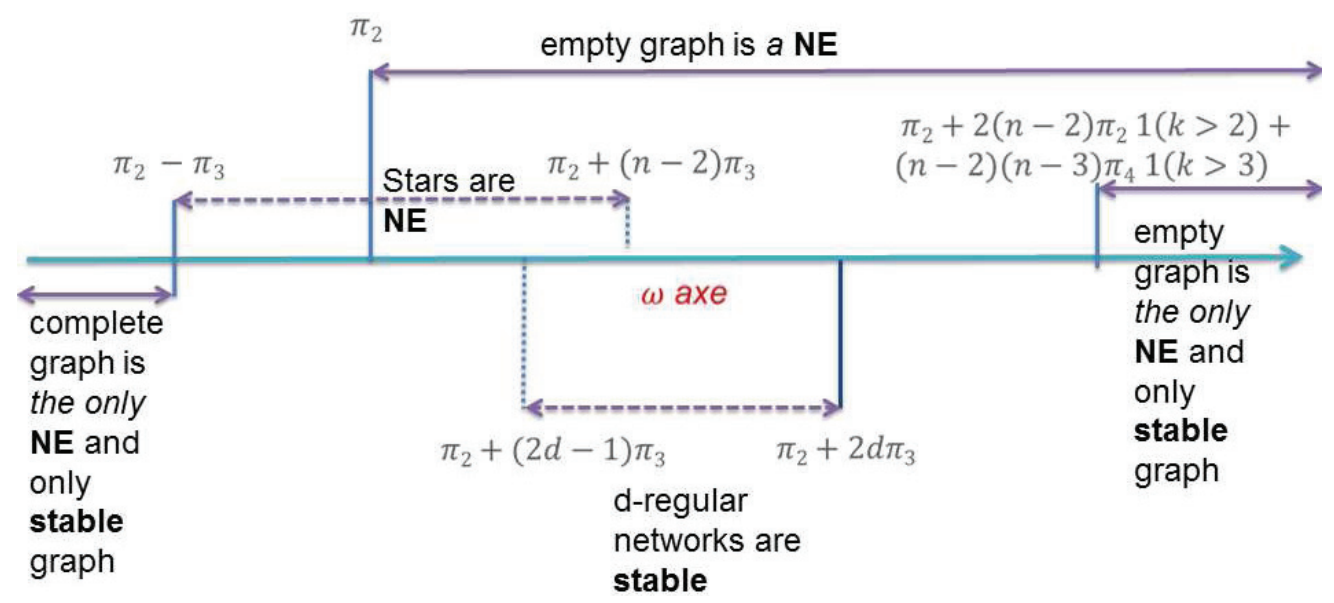




\section{OBTAINING CORE-PERIPHERY NETWORKS FROM STARS}

Real-life networks are seldom completely built from scratch. Rather, they usually arise from linking up pre-existing networks. In a market-environment with no central social planner, linking up is left to the incentives of individuals. Hence there is always the possibility that the linking, left to individualistic profit-maximising agents, would not result in an efficient network for the whole, even though taken individually each pre-existing network (which could be thought as, for example, national markets before the opening of their borders) was efficient. That is, the incentives of the agents in linking these different historical networks may not lead to an efficient network of the whole.

The notion of incentives is, in our framework, captured by the notion of stability and Nash equilibrium. Indeed, by definition in a strict Nash equilibrium no player would add and/or delete any set of links, since it would result in a lower payoff for him. In a strict stable network, no player would add a single new link nor delete a single existing link for the same reason. Now the problem of knowing if letting individual agents linking separated networks leads to an efficient network can be easily formalised in the following way: a certain number of efficient networks $H_{1}$, $H_{2}, \ldots, H_{k}$ are suddenly considered as the different disjoint parts of a (bigger) aggregate network $G$. Which structure will arise? Will this structure be efficient? Are there many different structures that can arise from such an operation or is the outcome predictable? Definition 24 allows to formalize the concept of an aggregate network obtained in a way that preserves its individual components in an endogeneous maner. Moreover, it provides a way to conceptualise the building up of a specific core-periphery network, which we show can be seen as resulting from the linking up of a set of disjoint stars.

Definition 24 A network $G$ has a partition $\left\{H_{i}, i \in I\right\}$ of subnetworks if, and only if, each $H_{i}$ is a subnetwork of $G$, and each node of $G$ belongs to a unique $H_{i}$. In other words:

$$
E\left(H_{i}\right) \subseteq E(G) \text { for all } i \in I \text { and } V(G)=\coprod_{i \in I} V\left(H_{i}\right)
$$

We see that asking $G$ to be stable, or to be a Nash equilibrium, is actually simply rephrasing the previous problem in the particular case where none of the "pre-existing" networks $H_{1}, H_{2}, \ldots, H_{k}$ has been modified - and thus all are still present as such in the resulting aggregate network. That is, "domestic" structures were preserved and linked in a way compatible with the individual incentives of each node. How does this linking occur? Does it lead to an efficient network for the whole without the need for coordination of the economic agents involved? Important to notice is that the preservation of the subnetworks $H_{1}, H_{2}, \ldots, H_{k}$ is not linked to sunk costs (costs to establish their links that were already paid for and hence less profitable to destroy), but follow the same general one-period game setting than the rest of the paper. In that sense the networks can be seen more as reflecting domestic agents" "habits" for paying for and using a domestic, local network, than as reflecting some legacy infrastructure already built. This makes the results of this subnetwork "preservation" and the study of the emerging core-periphery network that follows all the more striking.

A simple example where the answer is affirmative is for parameter constellations such that $w<\frac{g+c}{6}$. Indeed, by Proposition 17 we know that the unique Nash equilibrium network in that case is the complete network, and it is easily checked that it is also efficient. Now it can easily be show that taking the union of any number of complete networks will result in a global network which is also complete, hence also efficient for such constellations of parameters: since $w<\frac{g-c}{2}$ 
the union, at equilibrium, will be strongly connected. Because $w<\frac{g+c}{6}=\frac{g-c}{2}-\frac{g-2 c}{3}$ one can prove that transitivity holds at equilibrium, and conclude as in the proof of Proposition 17.

Theorem 26 and Theorem 27 focus on the case where all the graphs $H_{i}$ are complete stars (possibly of different sizes). These theorems provide a condition under which the supremacy of the centers of a star is in fact re-enforced by aggregation, since each leaf wants to send a link to the center of each foreign stars on top of maintaining the link towards its own domestic center, and do not link to other nodes than the centers. This proves that, for some constellation of parameters, aggregation of different markets can actually increase the power of the historical "oligopoly" (formed by the centers of the stars) instead of creating new, alternative intermediation chains. This also provides an example of stable core-periphery network under homogeneous cost assumption.

We then prove that, for a wide range of parameters, these resulting networks are Paretodominated by the complete star. In particular, they cannot be efficient. More precisely, we will prove that the leaves would always be better off in a complete star, while for the center $s_{i}$ of star $S_{i}$ this depends on a condition that interlinks the size of the network, the size of $S_{i}$ and the number of other stars.

Definition 25 Define a multistar as a network $G$ having a partition $\left\{S_{i}, i \in I\right\}$ of complete stars $S_{i}$ of center $s_{i}$ such that each of the node $v \notin\left\{s_{i}, i \in I\right\}$ is linked to precisely all the $\left\{s_{i}, i \in I\right\}$ and each of the center $s_{i}$ is linked to precisely all the nodes in $\left(S_{i} \backslash\left\{s_{i}\right\}\right) \cup\left\{s_{j}, j \in \Lambda\{i\}\right\}$. We then say that $G$ is a multistar with partition $\left\{S_{i}, i \in I\right\}$ of complete stars $S_{i}$. The leaves of the multistar $G$ are defined as the leaves of the $S_{i}, i \in I$.

Figure 6.

Representation of a multistar. Only all the links of a single leaf, the node $x$, are represented in the picture for clarity.

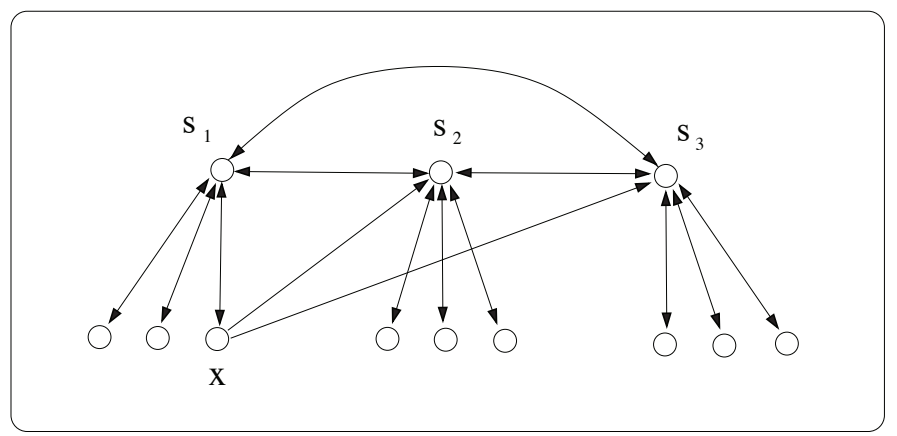

Multistars are thus a particular example of core-periphery

Theorem 26 Assume $g>2 c$ and $w<\frac{g+c}{6}$. Let $G$ be a strict Nash equilibrium. Then there is a threshold $d_{0} \leq \frac{3}{g-2 c}\left(w-\frac{g+c}{6}\right)+1$ such that, if $G$ has a partition $\left\{S_{i}, i \in I\right\}$ of complete stars, where $s_{i}$ denotes the center of star $S_{i}$, and such that, for all $i \in I$, we have:

$$
\left|\left(N^{+}\left(s_{i}\right) \cap S_{i}\right) \backslash \bigcup_{w \notin S_{i}} N^{+}(w)\right|>d_{0}
$$

then $G$ is a multistar with partition $\left\{S_{i}, i \in I\right\}$ of complete stars.

The proof of Theorem 26 is provided in Annex 6. 
In the more general case where chains of intermediaries of arbitrary length are allowed, a similar theorem can be proved (Theorem 27), with a different threshold:

Theorem 27 Assume $g>2 c$ and $w>\frac{g+c}{6}$. Let $G$ be a strict Nash equilibrium. Then there is a threshold $d_{0} \leq \frac{12}{g+c}\left(w-\frac{g+c}{6}\right)+1$ such that, if $G$ has a partition $\left\{S_{i}, i \in I\right\}$ of complete stars, where $s_{i}$ denotes the center of star $S_{i}$, and such that, for all $i \in I$, we have:

$$
\left|\left(N^{+}\left(s_{i}\right) \cap S_{i}\right) \backslash \bigcup_{w \in S_{i}} N^{+}(w)\right|>d_{0}
$$

then $G$ is the unique network such that 1) it contains the $\left\{S_{i}, i \in I\right\}$ as subnetworks, and 2) each of the node $v \notin\left\{s_{i}, i \in I\right\}$ is linked to precisely all the $\left\{s_{i}, i \in I\right\}$. 3) Each center $s_{i}$ is linked to precisely all the nodes in $S_{i} \cup\left\{s_{j}, j \in \Lambda\{i\}\right\}$.

The proof is similar to the one of Theorem 26 provided in the Annex, and thus omitted there.

Theorem 26 and Theorem 27 can be interpreted as follows: if each center has some large enough "private" market (larger than the $d_{0}$ threshold, which is not larger than the upper bound given by the theorem), that is, a set of participants which can only be accessed using the center as an intermediary, then all the other leaves will decide to link to those centers, and to those centers only. Theorem 26 and Theorem 27 thus considerably restrict the number of possible structures in equilibrium.

Conversely to Theorem 26, it can be proved that this unique possible structure is indeed a Nash equilibrium if we add the well known and usual necessary condition that $w \leq \frac{g-c}{2}+(n-2) \frac{g-2 c}{3}$, together with a sufficiently high degree for each center of the stars:

Proposition 28 Let $G$ be a multistar with partition $\left\{S_{i}, i \in I\right\}$ of complete stars $S_{i}$. Assume moreover than $d^{+}\left(s_{i}\right)-|I|+1>d_{0}$ for all $i \in I$, and that $\frac{g+c}{6}<w<\frac{g-c}{2}+(n-2) \frac{g-2 c}{3}$. Then $G$ is a strict Nash equilibrium.

The proof is provided in Annex 6.

\subsubsection{Efficiency comparison between stars and multistars}

The previous section showed how multistars can endogenously arise from the linking up of stars, with each star having its previous links preserved, as formalised in Definition 24. Assume $G$ is a multistar with partition $\left\{S_{i}, i \in I\right\}$ of complete stars $S_{i}$ of center $s_{i}$. When $d^{+}\left(s_{i}\right)-|I|+1>d_{0}$ for all $i$, and $\frac{g+c}{6}<w<\frac{g-c}{2}+(n-2) \frac{g-2 c}{3}$, we know by Proposition 18 and Proposition 28 that both the complete star and the multistar are strict Nash equilibrium. The question then arises to know which of the two networks is the most efficient from an aggregate perspective (social welfare). This has clear policy implications, if we remember that the multistar arises naturally as an equilibrium when the re-organisation of the network obtained from various disjoint stars is left to the individual, profit-maximising agents of each of the stars. We characterise parameter constellations for which, from an aggregate utility perspective, the star is always a more efficient network than a multistar.

Theorem 29 Let $h(c, w)=5 n / 4-1-(n-2) w / c$. Let $i$ be the closest positive number to $h(c, w)$. Then among all the multistars, the ones with precisely $i$ centers are the most efficient networks. 
The proof uses simple combinatorics arguments and the maximisation of the aggregate cost function. It is relegated to Annex 6. Theorem 29 has the obvious corollary:

\section{Corollary 30 If $h(c, w)<1.5$ then the star is more efficient than any other multistar.}

Theorem 29 indicates that the efficiency of a multistar can be non-monotonous in the number of centers of the multistar and has interesting consequences. When the cost for building a link $w$ increases, $h(c, w)$ decreases, hence multistars with more centers become less efficient compared to more concentrated structures. This is due to the larger number of edges that need to be built in a multistar compared to a star or to more concentrated multistar. When on the contrary the cost of using a link $c$ increases, less concentrated structures, that is, multistars with larger number of centers, become more attractive, as in particular the direct links between multistars allow for costreduction in inter-center trading.

To compare stars and multistars, we can also use the notion of Pareto-domination for unlabeled graphs. We will convene that a (unlabelled) network $G$ Pareto-dominates another (unlabelled) network $H$ if there is some way to relabel the vertices of $G$ such that each node in the relabelled network is better off than in $H^{5}$. A characterisation for stars to Pareto-dominate multistar in that sense is given in Theorem 34 in Annex 6.

Notice that the arising inefficiencies of multistars do not come from the fixed investments spent for building links, but lie uniquely in the new individual incentives for reshaping the network that take into account the a preferred local structure and thus are not enough to lead to an efficient resulting network. This finding has obvious policy implications, such as the potential benefits of the intervention of a "social planner" for re-arranging by itself the network or for acting as a catalyst for coordination among the different players, or for introducing incentives exogenous to the network that could help fostering a more efficient aggregate network.

\section{CONCLUSION}

In this paper we introduced a specific rule for sharing profits along intermediary chains, which entails natural yet apparently complex payoffs and strategic behaviour from the network agents. We analyzed different general network structures, and derived the conditions for those networks to be stable - that is, no individual in the network would be better off by adding or deleting a link it has some power on. We defined a notion of graph partition that translates the existence of substructures preserved yet incorporated in a larger, aggregate network and studied the case of a particular type of core-periphery structure that can be seen as resulting from linking up a set of disjoint stars. This structure, or multistar, is a core-periphery network that allows for different vertex degrees between the different centers which form its core, and we prove it is stable for a large set of parameter constellations without having to resort to heterogeneity assumptions. We derived conditions under which stable multistars are not efficient nor Paretoefficient. This emphasises the theoretical need, in some instances, for a "social planner" to rearrange the network or at least act as a catalyst in the new network formation, in particular in complex networks obtained by linking pre-existing networks, even when these smaller networks

5 It is easy to see how the notion of Pareto-domination for an unlabelled graphs may differ from the more common game theoretic Paretodomination. For example consider two identical complete stars of same size $G$ and $H$, and assume their center earns more than their leaves. Suppose also that node 1 is the name of the center of $G$ but the name of a leaf in $H$. Then $H$ does not Pareto-dominate $G$ as a labelled graph, since the node 1 is worse off in the network $H$ than in the network $G$. But $H$ does Pareto-dominate $G$ as an unlabelled graph: there is a way to re-labell its vertices so that to obtain a labelled graph which Pareto-dominates $H$ : any re-labelling where the center of $G$ has the same name as the center of $H$ will of course do. 
were, individually taken, efficient. Further work is needed to characterise, in this context, the whole set of efficient networks, as well as of stable networks and Nash equilibrium network, but this appears to be a difficult tasks, in particular when longer chains of intermediaries are allowed, because of the strategic considerations at play that take into account a larger portion of the topology of the network.

\section{References}

Bala V., Goyal S. (2000) A noncooperative model of network formation. Econometrica 68, no 5, pp. 1181-1229.

Babus, A., (2013) Endogenous Intermediation in over-the-counter markets. Imperial College London working paper. Belleflamme P., Bloch F. (2004) Market sharing agreements and collusive networks. International Economic Review 45 No 2, pp. 387-411.

Buskens, V., van de Rijt, A. (2008) Strategic network formation with structural holes. American Journal of Sociology 114, pp. 37-407.

Calvo-Armengol A., Jackson M.O. (2004) The effect of social networks on employment and inequality. American Economic Review 94, pp. 426-454.

Craig, B., von Peter, G. (2014) Interbank tiering and money center banks. Journal of Financial Intermediation (Forthcoming)

Veld D., van der Leij M., Hommes C. (2014) The formation of a core periphery structure in heterogeneous financial networks, Tinbergen Institute Discussion Paper TI 2014-098/II.

Dutta B., Ghosal S., Ray D. (2004) Farsighted network formation. Journal of Economic Theory 122, pp. 143-164.

Fricke, D., Lux, T. (2014) Core-periphery structure in the overnight money market: Evidence from the e-MID trading platform. Computational Economics (Forthcoming).

Gimbert J. (1999) On digraphs with unique walks of closed lengths between vertices. The Australasian Journal of Combinatorics 20, pp. 77-90.

Goyal S., Joshi S. (2003) Networks of collaboration in oligopoly. Games and Economic Behavior 43, pp. 57-85.

Goyal S., Vega-Redondo F. (2003) Network formation and social coordination. Working Papers 481, Queen Mary, University of London, School of Economics and Finance.

Jackson M.O. (1996) A strategic model of social and economic networks. Journal of Economic Theory 71, pp. 44-74.

Jackson M.O. (2002) The evolution of social and economic networks. Journal of Economic Theory 106, pp. 265-295.

Jackson M.O., Wolinsky A. (1996) A strategic model of economic and social networks. Journal of Economic Theory 71, pp. (1) 44-74.

Jackson M.O., Watts A. (2001) The existence of pair-wise stable networks. Seoul Journal of Economics 14, pp. 299-321.

Kleinberg, J., Suri, S., Tardos, E., Wexler, T. (2008) Strategic network formation with structural holes. Proceedings 9th ACM Conference on Electronic Commerce, pp. 480-489.

Matutes C., Padilla A.J. (1994) Shared ATM networks and banking competition. European Economic Review 38, pp. 1113-1138.

McAndrews J. J., Rob R. (1996) Shared ownership and pricing in a network switch. International Journal of Industrial Organization 14, pp. 727-745.

Siedlarek J. (2011) Intermediation in Networks, Nota di Lavoro. FEEM Working Paper 42.

Spence M. (1984) Cost reduction, competition, and industry performance. Econometrica 52 No 1, pp. 101-122.

Vickers J. (1995) Competition and regulation in vertically related markets. Review of Economic Studies 62, pp. 1-17.

Valletti T.M., Cambini C. (2005) Investments and network competition. The RAND Journal of Economics 36 No 2, pp. 446-467.

Volgelsang I. (2003) Price regulation of access to telecommunications networks. Journal of Economic Literature 4, pp. 830-862. 


\section{APPENDIX \\ Proofs of theorems}

Proposition 13 If $w<\frac{g-c}{2}$, then any stable network is strongly connected and its diameter satisfies $\Delta \leq \min \left(k_{0}-1,\left\lfloor\frac{\frac{g+c}{2}+w}{\frac{g+c}{2}-w} \mid\right)\right.$.If(i) $w<(g+c) \sum_{k=2}^{n} \frac{1}{k}-c$ and(ii) $k_{0}-1 \geq\left\lfloor\frac{\frac{g+c}{2}+w}{\frac{g+c}{2}-w} \mid\right.$, then the bound is optimal, with the directed circle $C$ of diameter $\Delta_{C}=\left\lfloor\frac{\frac{g+c}{2}+w}{\frac{g+c}{2}-w}\right\rfloor$ being a stable network for which the bound is reached.

Proof. We first prove that any stable network is strongly connected, and then derives the upperbound for its diameter. Let $G$ be a stable network and suppose it is not strongly connected. Let $A$ and $B$ be two nodes such that there exists no path going from $A$ to $B$. Building the edge $(A, B)$ would incur a cost of $w$ to node $A$ but an additional profit of at least $\frac{g-c}{2}$. Indeed, the trade with $B$ itself already provides $A$ with a profit of $\frac{g-c}{2}$, to which may be added other intermediation fees from the possible admissible paths using the newly created edge $(A, B)$. Since $w<\frac{g-c}{2}$ the node $A$ would be willing to build $(A, B)$. This contradicts the stability of the network $G$.

Consider a minimum path $P$ of length $\Delta, P=A_{0}, A_{1}, A_{2}, \ldots, A_{\Delta}$ and assume $\Delta \geq 2$ (the case where $\Delta \leq 1$ can be readily checked since for network with at least two nodes, $w<\frac{g-c}{2}$ implies $\Delta=1$ and $\frac{\frac{g+c}{2}+w}{\frac{g+c}{2}-w}>1$ since $w>0$, while by assumption $k_{0} \geq 2$.) Hence by minimality of $P$, $A_{0} A_{\Delta}$ is not an edge. Since $w<\frac{g-c}{2}, A_{0}$ would certainly be willing to pay for creating an edge towards $A_{\Delta}$ if it could not use the minimum path $A_{0}=A, A_{1}, A_{2}, \ldots, A_{\Delta}$ for carrying out trades with $A_{\Delta}$, in which case we would have a contradiction with the minimality of $P$. Stability thus implies that this path can be used, hence that its length $\Delta$ is at most $k_{0}-1$. Also, stability requires that the benefits derived by $A_{0}$ from trading with $A_{\Delta}$ by using this minimum path is no less than the benefits it would get if it chose to establish a direct link with $A_{\Delta}$, idem est that $\frac{g-\Delta c}{\Delta+1} \geq \frac{g-c}{2}-w$, which provides the condition $\Delta \leq \frac{\frac{g+c}{2}+w}{\frac{g+c}{2}-w}$. Hence $\Delta \leq \min \left(k_{0}-1, \frac{\frac{g+c}{2}+w}{\frac{g+c}{2}-w}\right)$.

To prove that the directed circle attain the bound under the conditions mentioned, just consider that the condition for any vertex not to delete its single link is that $w<\sum_{k=2}^{n} \pi_{i}=\sum_{k=2}^{n} \frac{g-(k-1) c}{k}=(g+c) \sum_{k=2}^{n} \frac{1}{k}-c$.

Notice in passing that another (weaker) necessary condition for the minimum path to be able to carry out trade is that $g-\Delta c>0$ ie $\Delta \leq g / c$. Hence the above results also contains the somehow simpler condition that $\Delta \leq g / c$.

Proposition 16 If $w>\frac{g-c}{2}+2(n-2) \frac{g-2 c}{3} 1_{\left(k_{0}>2\right)}+(n-2)(n-3) \frac{g-3 c}{4} 1_{\left(k_{0}>3\right)}=: l_{0}$, then the empty network is the only strict stable network (and the only strict Nash equilibrium). 
Proof. Suppose $g \leq 2 c$. Without loss of generality $k_{0} \leq 2$ and the assumption becomes that $w \geq \frac{g-c}{2}$. This implies that the direct gains for $A$ from establishing a link towards $B$ are $\frac{g-c}{2}-w<0$. Hence no node $A$ will ever choose to maintain a link. This results in a empty network.

Suppose $g>2 \mathrm{c}$ and that $w>l_{0}$. It is enough to show that $l_{0}$ is the maximal additional profit a node $A$ can hope for by establishing a link. Since the costs $w$ are superior to $l_{0}$ it will then follow that no node $A$ will ever want to maintain a link, and that the empty network is the only Nash equilibrium for such parameter constellations. To show that $l_{0}$ is an upper bound for the additional profits derived by establishing a single edge $A B$, we start from any graph $G$ and prove by successive reductions than the additional profit a node $A$ can derive by establishing an edge $A B$ cannot be more than the additional profit node $A$ would derive from edge $A B$ in a particular graph $K_{0}$, whose structure is known and in which $A$ derives from $A B$ an additional profit less or equal to $l_{0}$.

Additional profits for $A$ derived from establishing a new edge $A B$ stems from:

- $\quad$ the direct trade with $B$, which amounts to at most $\frac{g-c}{2}$ - this upper-bound corresponding to the case where there were no admissible path going from $A$ to $B$ prior to the establishment of the link $(A, B)$.

- $\quad$ the profits from trades involving more than two intermediaries and using a chain $P$ of intermediaries which finishes with the edge $A B$. Assume such chain contains more than four intermediaries, and let $P=: C \ldots D A B$. Then $A$ derives less profits in such a graph from trades $C B$ than it would if $C$ was a in-neighbours of $A$, the reason being it has to share the profits from trade $C B$ with more intermediaries (for example, with $D$ ). Hence $A$ obtains no less profits in the graph $G^{\prime}$ obtained from $G$ by adding all edges pointing towards $A$, than in $G$. These profits amounts to a profit from these trades of less or equal to $(n-2) \frac{g-2 c}{3}$ in $G^{\prime}$.

- $\quad$ the profits from trades involving more than two intermediaries and using a chain $P$ of intermediaries which starts with the edge $A B$. Similarly, $A$ obtains no less profits in the graph $G^{\prime \prime}$ obtained from $G^{\prime}$ by adding all edges starting from the node $B$, than in $G^{\prime}$. These profits amounts to a profit from these trades of less or equal to $(n-2) \frac{g-2 c}{3}$ in $G^{\prime \prime}$.

- $\quad$ the profits from trades involving more than three intermediaries and using a chain $P$ of intermediaries in which both $A$ and $B$ are strict intermediaries. Note these profits can only be positive if $k_{0}>3$. Also, these profits are maximal when no node in $V(G) \backslash\{A, B\}$ is linked to another node of $V(G) \backslash\{A, B\}$. Indeed, in such a configuration any node in $V(G) \backslash\{A, B\}$ has to use the node $A$ to carry out a trade with another node from $V(G) \backslash\{A, B\}$. Hence, $A$ obtains no less profits in the graph $G_{0}$ obtained from $G^{\prime \prime}$ by deleting all edges between two nodes in $V(G) \backslash\{A, B\}$ than in $G^{\prime \prime}$. These profits amounts to $(n-2)(n-3) \frac{g-3 c}{4} 1_{\left(k_{0}>3\right)}$ in $G_{0}$.

It results from the above that $\frac{g-c}{2}+2(n-2) \frac{g-2 c}{3}+(n-2)(n-3) \frac{g-3 c}{4} 1_{\left(k_{0}>3\right)}$ is the additional profit derived by node $A$ by establishing the link $A B$ in the graph $K_{0}$, and that it is an upper-bound for the additional profit derived by node $A$ by establishing the link $A B$ in the initial graph $G$. Since this holds for all $G$, we have a general upper-bound. 
Proposition 19: Assume $g>2 c$. Then stable complete stars are also Nash equilibria.

Proof. Let $G$ be a complete stable star.

By symmetry of the network, the center's payoff depends uniquely on the number of links it creates towards the other nodes. Assume it creates $\mathrm{k}$ links, and let $\mathrm{f}$ be its payoff. Then

$$
f(k)=\{-k w\}+\left\{k\left(\frac{g-c}{2}\right)+(n-1)\left(\frac{g-c}{2}\right)\right\}+\left\{k(k-1)\left(\frac{g-2 c}{3}\right)+(n-1-k) k\left(\frac{g-2 c}{3}\right)\right\}
$$

Indeed, the first term between brackets is the costs of creating the links, the second term between brackets the profits from direct transactions and the third term between brackets the profits from intermediation. $f(k)$ can be rewritten as:

$$
f(k)=k\left(-w+\left(\frac{g-c}{2}\right)+(n-2)\left(\frac{g-3 c}{3}\right)\right)+(n-1)\left(\frac{g-c}{2}\right)
$$

Since $-w+\frac{g-c}{2}+(n-2)\left(\frac{g-2 c}{3}\right) \geq 0$ the maximum is obtained for the maximum, value of $k$ that is, $n-1$, which means creating all possible links. Note that, in the above reasoning, instead of computing the total profits, we could have only computed the additional profits obtained by establishing $k$ links, that is $k\left(-w+\frac{g-c}{2}+(n-2)\left(\frac{g-2 c}{3}\right)\right)$. Because $-w+\frac{g-c}{2}+(n-2)\left(\frac{g-2 c}{3}\right) \geq 0$ the maximum is obtained for the maximum value of $k$, which is $n-1$, and we conclude similarly. This approach being somehow simpler, we proceed in this fashion for the leaves, computing the additional benefits of having a given number of links in each case.

For computing one of the leaf $A$ 's best-response we will distinguish two cases:

In the first case the leaf $A$ plays a strategy where it builds a link towards the center and $k$ other links, for $k \in\{0,1, \ldots, n-2\}$. Its additional profits from building $k$ edges towards other leaves is: $k\left(-w+\frac{g-c}{2}-\frac{g-2 c}{3}\right)$. Because $\frac{g+c}{6}-w \leq 0$, the maximum is obtained for $k=0$, which means building no link except towards the center.

In the second case it does not link to the center but to $k$ other links, for $k \in\{0,1, \ldots, n-2\}$. If $k \geq 1$ then the additional profit obtained by $A$ if it severs all existing links and connects to the center instead is: $(k-1) w-(k-1) \frac{g-c}{2}+(k-1) \frac{g-2 c}{3}+(n-2-k)\left(\frac{g-2 c}{3}-\frac{g-3 c}{4} 1_{\left(k_{0}>3\right)}\right)$. Indeed, the first term represents the costs saved from having to pay only for a single link instead of $k$ links, the second the loss of profits from direct trades towards other nodes, the third the compensating gains from indirect trade steping up to replace the previously direct trades, and the last term the gains in what was before, and still is, indirect trade $\left(1\left(k_{0}>3\right)\right.$ is 1 if $k_{0}>3$ and 0 otherwise). This amounts to $(k-1)\left(w-\frac{g+c}{6}\right)+(n-2-k)\left(\frac{g-2 c}{3}-\frac{g-3 c}{4} 1_{\left(k_{0}>3\right)}\right)$, a non-negative quantity since $w-\frac{g+c}{6} \geq 0$ and $\frac{g-2 c}{3}-\frac{g-3 c}{4} 1_{\left(k_{0}>3\right)}>0$. Hence strategies sending edges towards leaves and not towards the center are dominated by the strategy of just linking to the center.

The only check left is the suboptimality of not building any edge. Building a single edge towards the center would bring an additional profit of $-w+\frac{g-c}{2}+(n-2)\left(\frac{g-2 c}{3}\right) \geq 0$, where $\frac{g-c}{2}$ stems from direct trade of the leaf $A$ with the center and $(n-2)\left(\frac{g-2 c}{3}\right)$ from indirect trades starting at $A$. This concludes the proof. 
Proposition 23: Assume $g>2 c$ Then a $d$-regular network is stable if, and only if,

$$
2 d\left(\frac{g-2 c}{3}\right)+\left(\frac{g+c}{6}\right) \leq w \leq \frac{g-c}{2}+2 d\left(\frac{g-2 c}{3}\right)
$$

The proof of Proposition 23 relies both on Theorem 22 and on the following Lemma:

Lemma 31 Suppose $g>2 c$. Suppose $N^{+}(A) \cap N^{-}(B) \neq \varnothing$. Then node A earns, by creating the link $(A, B)$, an additional profit of

$$
\left(\left|N^{-}(A)\right|-\left|N^{-}(A) \cap N^{-}(B)\right|+\left|N^{+}(B)\right|-\left|N^{+}(A) \cap N^{+}(B)\right|\right)\left(\frac{g-2 c}{3}\right)+\frac{g+c}{6}-w
$$

Proof. The additional profits of creating such a link are (see Figure 7):

1) those derived from intermediating transactions using chains of type $D A B$ where $D$ is any node in $N^{-}(A)$ but not in $N^{-}(B):\left(\left|N^{-}(A)\right|-\left|N^{-}(A) \cap N^{-}(B)\right|\right)\left(\frac{g-2 c}{3}\right)$,

2) those derived from intermediating transactions using chains of type $A B C$ where $C$ is any node in $N^{+}(B)$ but not in $N^{+}(A):\left(\left|N^{+}(B)\right|-\left|N^{+}(A) \cap N^{-}(B)\right|\right)\left(\frac{g-2 c}{3}\right)$,

3) the additional profit $\frac{g-c}{2}-\frac{g-2 c}{3}=\frac{g+c}{6}$ of carrying out directly trades $(A, B)$ instead of using one strict intermediary.

The costs are $w$. The result follows

\section{Figure 7.}

Illustration of the different chains of intermediaries alluded to in the proof

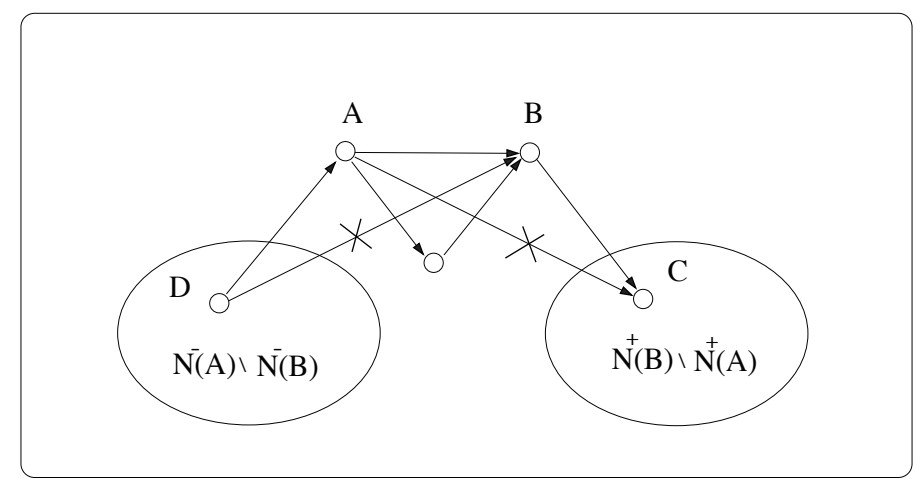

We can now proceed to prove that the condition of Proposition 23 is necessary and sufficient:

Suficiency: Suppose $(A, B)$ is not an edge. Since $G$ is a uninetwork we thus have a node $C$ such that the path $A C B$ allows to settle trades of type $(A, B)$, and $C \in N^{+}(A) \cap N^{-}(B) \neq \varnothing$. We have, since $\left|N^{-}(A)\right|=d=\left|N^{+}(B)\right|$ :

$\left(d-\left|N^{-}(A) \cap N^{-}(B)\right|+d-\left|N^{+}(A) \cap N^{+}(B)\right|\right)\left(\frac{g-2 c}{3}\right)+\frac{g+c}{6} \leq\left(\frac{g-2 c}{3}\right)(2 d)+\left(\frac{g+c}{6}\right) \leq w$

Hence by Lemma 31 node $A$ does not want to create this edge.

Suppose $(A, B)$ is an edge. Deleting it would incur a gain of precisely $w-\frac{g-c}{2}-d\left(\frac{g-2 c}{3}\right) \leq 0$, since there is no other path than $(A, B)$ that can be used by $A$ to trade with $B$ nor other path than $A B C$ which can be used to trade with $C$ for any $C \in N^{+}(B)$. Hence $A$ would be worse off deleting $(A, B)$. Hence the condition is sufficient. 
Necessity: The most important part of the proof is indeed to establish the necessity of the condition, as it will allow us to conclude the network we are looking for does not exist. Indeed, for such a network to be stable it is necessary that no node $A$ wants to create a link towards $B$ for any $A B \notin E(G)$. Let $A \in V(G)$. Let $A^{\prime}$ be the unique node of $G$ which is both an in-neighboor and an out-neighboor of $A$. The existence and unicity of $A^{\prime}$ is assured by Theorem 22 and the definition of a line network of a complete network (Definition 21). Since $d \geq 2$ there exists $v$ in $N^{-}(A) \backslash\left\{A^{\prime}\right\}$. Hence $v A \in E(G)$.

Suppose $N^{-}(A) \cap N^{-}(v) \neq \varnothing$. Then there exists $z$ in $N^{-}(A) \cap N^{-}(v)$ and $z v A$ and $z A$ are two distinct paths going from $z$ to $A$, a contradiction with the definition of uninetwork. Hence $N^{-}(A) \cap N^{-}(v)=\varnothing$. Similarly, we can show that $N^{+}(A) \cap N^{+}(v)=\varnothing$. Since we suppose that the network $G$ is stable, and that $A v \notin E(G)$, node $A$ should not want to create an edge towards $v$. Applying then Lemma 31 to the pair $(A, v)$ yields the condition $2 d\left(\frac{g-2 c}{3}\right)+\left(\frac{g+c}{6}\right) \leq w$.

For the other part of the inequality, notice again that if $(A, B)$ is an edge in the network, deleting it would incur a gain of precisely $w-\frac{g-c}{2}-2 d\left(\frac{g-2 c}{3}\right)$, since there is no other path than $(A, B)$ that can be used by $A$ to trade with $B$ nor other path than $A B C$ which can be used to trade with $C$ for any $C \in N^{+}(B)$, nor other path than $D A B$ which can be used by $D \in N^{-}(A)$ to trade with $B$. Assuming this quantity to be nonpositive is thus necessary. This concludes the proof of the necessity of the condition.

Theorem 26: Assume $g>2 c$ and $w>\frac{g+c}{6}$. Let $G$ be a strict Nash equilibrium. Then there is a threshold $d_{0} \leq \frac{3}{g-2 c}\left(w-\frac{g+c}{6}\right)+1$ such that, if $G$ has a partition $\left\{S_{i}, i \in I\right\}$ of complete stars, where $s_{i}$ denotes the center of $\operatorname{star} S_{i}$, and such that, for all $i \in I$, we have:

$$
\left|\left(N^{+}\left(s_{i}\right) \cap S_{i}\right) \backslash \bigcup_{w \notin S_{i}} N^{+}(w)\right|>d_{0}
$$

then $G$ is a multistar with partition $\left\{S_{i}, i \in I\right\}$ of complete stars.

Proof. Let $S_{i}:=N^{+}\left(s_{i}\right) \backslash \bigcup_{w \notin S_{i}} N^{+}(w)$ be the set of vertices of $S_{i}$ that can only be reached
through $s_{i^{*}}$

Let $j \in I$. Let $v \in V(G) \backslash\left\{s_{j}\right\}$.

a) Assume first that $v \notin S_{j}$. If $v$ did not had a link towards $s_{j}$, then creating it would provide $v$ with an additional profit of at least

$$
-w+\frac{g-c}{2}-\frac{g-2 c}{3}+\mid S_{j}\left(\frac{g-2 c}{3}\right)
$$

Indeed, $w$ is the cost of establishing a new link towards $s_{j}, \frac{g-c}{2}-\frac{g-2 c}{3}=\frac{g+c}{6}$ is a lower bound for the additional gains from direct trade with $s_{j}$ and the last term represents the additional gains from intermediating trade with the $\left|S_{j}\right|$ nodes that can only be accessed only through $s_{j}$. It is thus a lower bound for the additional (strict) intermediation profits.

b) If $v \in S_{j}$ then the additional profit of creating a link to $s_{j}$ will be at least

$$
-w+\frac{g-c}{2}-\frac{g-2 c}{3}+\left(\left|S_{j}\right|-1\right)\left(\frac{g-2 c}{3}\right)
$$


This lower bound is lower than in a) because $v$ does not trade with itself in the model. This last lower bound, and hence both lower bounds, are positive as soon as $\left|S_{i}\right|>\frac{3}{g-2 c}\left(w-\frac{g+c}{6}\right)+1=: d_{0}$ which is the case by assumption. This contradicts the stability of a Nash equilibrium. Hence every $v \in V(G) \backslash\left\{s_{j}\right\}$ has a link to the center $s_{j}$ of $S_{j}$, and this result holds for all $j \in I$.

c) Let $v \in V(G) \backslash\left\{s_{i}, i \in I\right\}$. Let $k_{i}$ be the number of links that $v$ built towards $V\left(S_{i}\right)$. By the above, we already know that $v$ is linked to $s_{i} \in S_{i}$, hence $k_{i} \geq 1$. Assume by contradiction that $k_{i}>1$ for at least one $i \in I$. Then $\sum_{i \in I} k_{i}-|I|>0$. We can write the additional profit of node $v$ derived by these extra-edges as a function of the $k_{i}$ :

$-w\left(\sum_{i \in I} k_{i}-|I|\right)+\left(\sum_{i \in I} k_{i}-|I|\right)\left(\frac{g-c}{2}-\frac{g-2 c}{3}\right)=\left(\sum_{i \in I} k_{i}-|I|\right)\left(-w+\frac{g+c}{6}\right)$

which is strictly negative since $\sum_{i \in I} k_{i}-|I|>0$ and $-w+\frac{g+c}{6}<0$, a contradiction with $G$ Nash equilibrium. Hence $k_{i}=1$ for all $i \in I$ and $v$ sends only a single link towards each star $S_{i}$, which is directed towards the center $s_{i}$ of $S_{i}$.

d) Let now $i_{0} \in I$ and consider si0. The same argument as in (c), where for $i \in \Lambda\left\{i_{0}\right\}$ we define $k_{i}$ to be the number of links that $s_{i 0}$ built towards $V\left(S_{i}\right)$, can be used by considering $\sum_{i \in I \backslash\left\{i_{0}\right\}} k_{i}$ instead of $\sum_{i \in I} k_{i}$, and $\Lambda\left\{i_{0}\right\}$ instead of $I$. This concludes the proof.

Figure 8.

Illustration of the proof

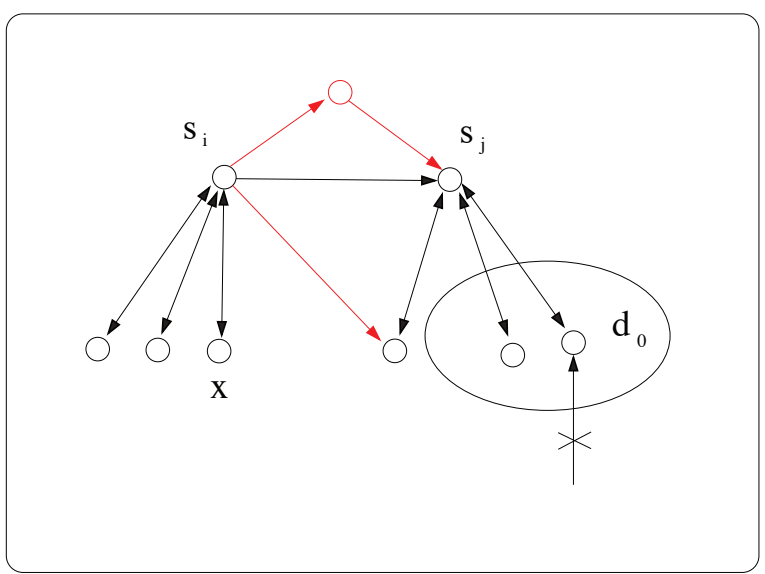

Conversely, it can be checked that this unique possible structure is indeed a Nash equilibrium when we had the now well-known necessary condition that $w \leq \frac{g-c}{2}+(n-2) \frac{g-2 c}{3}$, together with a sufficiently high degree for each center of the stars.

Proposition 28: Let $G$ be a multistar with partition $\left\{S_{i}, i \in I\right\}$ of complete stars $S_{i}$. Assume moreover than $d^{+}\left(s_{i}\right)-|I|+1>d_{0}$ for all $i \in I$, and that $\frac{g+c}{6}<w<\frac{g-c}{2}+(n-2) \frac{g-2 c}{3}$. Then $G$ is a strict Nash equilibrium.

Proof. The condition $d^{+}\left(s_{i}\right)-(|I|-1)>d_{0}$ is precisely the condition $(C)$ of the previous theorem once the overall structure has been deduced. The same arguments thus allow to prove that, given the other nodes strategies, each node strategy is a best-response among the strategies where the centers of the stars do maintain the links towards their leaves. More precisely, the additional benefit of the center $s_{i}$ of forming $k$ links towards its $d^{+}\left(s_{i}\right)-(|I|-1)$ leaves is, assuming other nodes do not deviate from the strategy profile: 


$$
\left(-w+\frac{g-c}{2}\right) k+(k(k-1)+k(n-1-k)) \frac{g-2 c}{3}
$$

Indeed, $-w k$ is the cost of maintaining these links, $\frac{g-c}{2} k$ the additional profit from direct trade, $k(k-1) \frac{g-2 c}{3}$ the intermediation profits from being the strict intermediary of trade between vertices both in $N^{+}\left(s_{i}\right) \cap S_{i}$ and $k(n-1-k) \frac{g-2 c}{3}$ the intermediation profits from nodes outside $S_{i}$ towards nodes in $N^{+}\left(s_{i}\right) \cap S_{i}$. This amounts to

$$
k\left(-w+\frac{g-c}{2}+(n-2) \frac{g-2 c}{3}\right)
$$

This additional benefit is increasing with the number of links created towards the leaves if, and only if, $w<\frac{g-c}{2}+(n-2) \frac{g-2 c}{3}$. By assumptions this condition is satisfied. Hence each center maintains a link towards each of its leaves at equilibrium.

Creating links towards leaves of other stars would result in a additional profit of $-w+\frac{g+c}{6}<0$ for the center $s_{i}$. Hence no center will maintain such links at equilibrium.

Any vertex (center or leaf) not in $S_{j}$ linking towards the center $s_{j}$ of another star $S_{j}$ will benefit from an additional profit of at least

$$
-w+\frac{g-c}{2}-\frac{g-2 c}{3}+\mid S_{j}\left(\frac{g-2 c}{3}\right)
$$

while any vertex in $S_{j}:=N^{+}\left(s_{j}\right) \backslash \bigcup_{w \notin S_{j}} N^{+}(w)$ linking towards the center $s_{j}$ of star $S_{j}$ will benefit from an additional profit of at least

$$
-w+\frac{g-c}{2}-\frac{g-2 c}{3}+\left(\left|S_{j}\right|-1\right)\left(\frac{g-2 c}{3}\right)
$$

The definition of $l_{0}$ insures doing so brings additional benefits.

We are only left with checking that linking directly to another leaf is not beneficial for a leaf, which is true since $-w+\frac{g+c}{6}<0$. We can thus conclude that the network $G$ is a Nash equilibrium.

Remark 32 We suspect the bound $d_{0}$ of Theorem 26 and Theorem 27 not to be optimal, since it is increasing with $w$, which is contrary to the intuition that higher costs of building edges would make intermediation through an oligopolistic more profitable. Hence, there may be a better bound than the one given by the above theorem.

Remark 33 In case $3 c<g$ which is the least to assume in the 4 intermediaries case, the upper bound for $d_{0}$ is lower (thus better) in Theorem 26 than in Theorem 27.

Theorem 29: Let $h(c, w)=5 n / 4-1-(n-2) w / c$. Let $i$ be the closest positive number to $h(c, w)$. Then among all the multistars, the ones with precisely $i$ centers are the most efficient networks.

Proof. First notice that since all the latent profits are realised, aggregate efficiency ranking can be obtained by simply inverting the ranking of the costs of the links: the parameter $g$ will thus drop from all the conditions derived here.

Simple combinatorics arguments allow to show that the number of edges in a multistar whose set of centers is $I$ is simply $n+|I|(n-2)$. Each of this edge cost w to build. 
In a multistar there are $(n-|I|) \cdot|I|+(n-|I|)+|I|(|I|-1)=|I|(n-2)+n$ direct trades. This is because there are $(n-|I|) .|I|$ direct trades from a leave to a center, $(n-|I|)$ direct trades from a center to a leaf (as there is a unique edge from $I$ to any of the $n-|I|$ leaves), and $|I|(|I|-1)$ edges linking the different centers of the multistar. Each of the direct trade is realised at a cost $c$.

In a multistar there are $(n-|I|) .(n-|I|-1)$ indirect trades, which follow routes of length 2. This is because each leaf indirectly trade with each other leaf of the multistar. Each of the indirect trade costs $2 c$.

Hence the total costs associated to a multistar with set of centers $I$ is:

$$
(n+|I|(n-2)) w+(|I|(n-2)+n) c+(n-|I|) \cdot(n-|I|-1) 2 c
$$

Define $f(x)=(n+x(n-2)) w+(x(n-2)+n) c+(n-x) \cdot(n-x-1) 2 c . f$ is a polynomial of degree 2 whose minimum is attained in $h(c, w)$, hence the result.

Theorem 34 Assume $c<w$. Let $G$ be a multistar with partition $\{S i, i \in I\}$ of complete stars $S_{i}$ of center $s_{i}$ and let $j$ be such that $\left|S_{j}\right|=\max \left(\left|S_{i}\right|, i \in I\right)$. Then a star with the same number of vertices as the multistar $G$ Pareto-dominates $G$ as soon as, for all $i \neq j$, we have $6(g-c-w)\left(|I|+\left|S_{i}\right|-2\right)$ $+3(g-c)\left(n-|I|-\left|S_{i}\right|-1\right)+2(g-2 c)\left\{-2(n-2)+(|S i|-1)\left(2 n-\left|S_{i}\right|-i-1\right)\right\}>g-c+$ $2(n-2) g-2 c 3$; and $\left(\left|S_{j}\right|-1\right)\left(n-\mid S_{j}\right)(g-2 c)>3\left(n-\left|S_{j}\right|-j+2\right) w$.

Proof. Leaves are better off in a star than in a multistar since in a multistar each leaf does $|I|-1$ more direct trades than in a star which save the transaction costs of $c(|I|-1)$ but building those additional links are paid by the leaf concerned hence a cost of $w(|I|-1)$. This results in a net additional cost of $(w-c)(|I|-1)>0$ in a multistar compared to the star, for the same profits being realised.

The center of a star is better than one of the center of the multistar $s_{i}$ if, and only if, $\left(\left|S_{i}\right|-1\right)\left(n-\left|S_{i}\right|\right)(g-2 c) / 3>\left(n-\left|S_{i}\right|-I+2\right) w$. Indeed using simple combinatoric arguments, we see that the center of the multistar, compared to the center of a star: (a) misses $\left(\left|S_{i}\right|-1\right)\left(n-\left|S_{i}\right|\right)$ paths of length 2 which realise profits from one leaf of their star $S_{i}$ to leaves not in $S_{i}$; (b) it builds $\left(n-\left(\left|S_{i}\right|-1\right)-(|I|-1)\right)$ less edges than the center of a star hence a saving of $\left(n-\left|S_{i}\right|-i+2\right) w$. Writing that profit losses are higher than cost cutting benefits is equivalent to the second condition.

The profit of a center of a star $s_{i}$ is the sum of the cost of (a) its $|I|+\left|S_{i}\right|-2$ edges: $-w\left(|I|+\left|S_{i}\right|-2\right)$, (b) the profits from direct trades to and from the $|I|-1$ other centers and its $\left|S_{i}\right|-1$ leaves: 2. $\left(|I|+\left|S_{i}\right|-2\right) \frac{g-c}{2}$, (c) the direct profit from trades from leaves outside $S_{i}:\left(n-\left|S_{i}\right|-|I|+1\right) \frac{g-c}{2}$, and (d) the indirect profits. The indirect profits can be further decomposed into: (i) the indirect profits from intra- $S_{i}$ trades: $\left(\left|S_{i}\right|-1\right)\left(\left|S_{i}\right|-2\right) \frac{g-2 c}{3}$, (ii) from trades from outside $S_{i}$ to a leaf in $S_{i}:\left(n-\left|S_{i}\right|\right)\left(\left|S_{i}\right|-1\right) \frac{g-2 c}{3}$, and (iii) from trades from a leaf in $S_{i}$ to a node outside: $\left(\left|S_{i}\right|-1\right)\left(n-\left|S_{i}\right|-|I|+1\right) \frac{g-2 c}{3}$. This results in an overall profit of

$$
\begin{aligned}
(g-c-w)\left(|I|+\left|S_{i}\right|-2\right)+ & \frac{g-c}{2}\left(n-|I|-\left|S_{i}\right|+1\right)+\frac{g-2 c}{3}\left(\left(\left|S_{i}\right|-1\right)\left(\left|S_{i}\right|-2\right)+\right. \\
& +\left(\left|S_{i}\right|-1\right)\left(2 n-2\left|S_{i}\right|-|I|+1\right)
\end{aligned}
$$

Writing that those profits are lower than those of the leaf of star is equivalent to the first condition.

When the first condition holds for all $i \neq j$, all the centers of a multistar with the possible exception of $s_{j}$ (one of the center eaning the most profits) earn less profits in a multistar than the leaves of a simple star. Under the second condition we have that the center of the star earns more than $s_{j}$. Since the leaves are better off in a star than being leaves in a multistar anyway, any 
relabelling of the centers of one of the center of the multistar which earns the most into the center of the star, and of the other centers into the name of simple leaves of the star, shows that the star Pareto-dominate the multistar.

Remark 35 The profit of a leaf $x$ of the multistar $G$ in $S_{i}$ is $-w|I|+\frac{g-c}{2}|I|+2 \frac{g-2 c}{3}(n-|I|-1)$ $+\frac{g-c}{2} 2+\frac{g-2 c}{3}(|I|-1)$. Indeed, $-w|I|$ is the cost of the leaf $x$ to maintain a link towards each of the centers, $\frac{g-c}{2}|I|$ the benefits from direct trade towards those centers, $2 \frac{g-2 c}{3}(n-|I|-1)$ the benefits of trading to and from the $n-|I|-1$ other leaves of the multistar, $\frac{g-c}{2}$ the benefits from direct trade from $s_{i}$ and $\frac{g-2 c}{3}(|I|-1)$ for indirect trades from the $s_{j}$ with $j \neq i$ to $x$. This can be rewritten as $|I|\left(-w+\frac{g-c}{2}-\frac{g-2 c}{3}\right)+\frac{g-c}{2}+(2 n-3) \frac{g-2 c}{3}$. Since $-w+\frac{g-c}{2}-\frac{g-2 c}{3}$ $=-w+\frac{g+c}{6}<0$, the lower $|I|$, the higher the profits for the leaf $x$. Economically, this expresses its preferences to only have to pay for one single link to get access to the whole network, in contrast to having to establish a link towards each center of a multistar. Note that, of course, by plugging $|I|=1$ we find again the profit of a star of size $n$. 